\title{
Calibration of CFUL01 fission chambers in the standard neutron fields of the BR1 reactor at SCK CEN
}

\author{
Anatoly Kochetkov, Antonín Krása, Luc Borms, Edouard Malambu, Guido Vittiglio, Jan Wagemans \\ and Jeroen Willems \\ SCK CEN, Belgian Nuclear Research Centre, Mol, Belgium \\ anatoly.kochetkov@sckcen.be
}

\begin{abstract}
Recent subcritical VENUS-F experiments showed that fission chambers with a threshold deposit like $U$ 238 can essentially improve the on-line sub-criticality measurments with the beam interruption method, which is currently supposed to be the main method for the ADS MYRRHA. To suppress the uncertainty caused by fissions in the U-235 impurities, the fraction of $U-235$ in the $U$ deposit should be accurately known. Three PHOTONIS CFUL01 type fission chambers with U-238 deposit were purchased for sub-critical experiments in the VENUS-F reactor. To verify the purity of their deposits, the effective U-235 masses were measured in the empty cavity of the BR1 reactor with a wellknown thermal neutron spectrum. It turned out that the measured effective U-235 mass in two fission chambers is lower than the declared mass (as it should be), but this is not the case for the third fission chamber. Then, the effective $U$ 238 mass in these FCs was measured in the well-known fast spectrum of the MARK-III convertor in the BR1 reactor. Finally, the isotopic composition was obtained and it was found that the purity of two CFUL01 FCs is in agreement with the values declared in the certificates but it is not the case for the third fission chamber. As the length of the deposit is bigger than the length of the MARK-III convertor, necessary corrections were calculated with MCNP. The developed procedure using the BR1 standard irradiation fields can be applied for calibration and impurity determination of large fission chambers.
\end{abstract}

Keywords - sub-critical monitoring, U-238 fission chambers, isotopic composition determination in BR1 neutron fields

\section{INTRODUCTION}

$\mathrm{T}$ HE FREYA FP7 project results show that the choice of the detector deposit and position in the core or reflector chosen for the reactor instrumentation can make reactivity monitoring easier or more difficult. Preliminary results show that fission chambers containing a U-238 deposit with a low U-235 content can be advantageously used for ADS sub-criticality measurements by applying the beam interruption method since they bring more flexibility as far as the duration of the necessary beam interruption is concerned [1].

Three PHOTONIS fission chambers of CFUL01 type with U-238 deposit were used for the sub-criticality measurements in a VENUS-F sub-critical core coupled with the GENEPI-III accelerator to simulate the ADS principle. The U-238 purity of the FCs according to the certificates is summarized in Table I.
The total masses of the deposits as well as the mass fractions in the certificates are presented. No information on the uncertainties is available.

TABLE I

THE PHOTONIS CERTIFICATE DATA OF THE CFUL01 FISSION CHAMBERS WITH U-238 DEPOSIT.

\begin{tabular}{|l|l|l|l|}
\hline \hline \multicolumn{1}{|c|}{ Fission chamber ID \# } & \multicolumn{1}{|c|}{673} & \multicolumn{1}{c|}{676} & \multicolumn{1}{|c|}{677} \\
\hline Total deposit mass, $g$ & 1.00 & 1.15 & 1.15 \\
U-238 wt \% & 99.964 & 99.9983 & 99.797 \\
U-235 wt \% & 0.035 & 0.0012 & 0.2016 \\
\hline \hline
\end{tabular}

To verify the purity (U-235 content) in the deposits of these FCs, the effective U-235 and U-238 masses were measured in the standard thermal and fast neutron fields of the BR1 reactor $[2,3]$. These fields are normally used to calibrate miniature fission chambers (typically 2-4 $\mathrm{mm}$ in diameter and 10-25 mm in length). Calibration of large fission chambers with $10 \times$ bigger dimensions represents a challenge.

\section{EFFECTIVE MASSES DETERMINATION}

The effective masses of U-235 ( $\left.\mathrm{M}_{\text {eff }}{ }^{235}\right)$ of CFUL01 FCs were measured in the thermal flux in the empty cavity while the effective masses of $\mathrm{U}-238\left(\mathrm{M}_{\mathrm{eff}}{ }^{238}\right)$ were measured in the fast flux of the MARK-III convertor in the BR1 reactor.

The effective masses were obtained using standard procedures [4]:

$$
M_{\text {eff }}=\frac{A}{6.02 \times 10^{23}} \times \frac{C_{F C}}{\Phi \times \bar{\sigma}_{e q}}
$$

where $A$ is the molar mass of a deposit, $C_{F C}$ is the count rate of a FC with $M_{\text {eff }}$ to be determined, $\Phi$ is the neutron flux in MARK-III or empty cavity at the position of the deposit, and $\bar{\sigma}_{e q}$ is the spectrum averaged fission cross-section (JEFF-3.1.1 [5]) of the deposit material (U-235 or U-238 with impurities in the center of the cavity or in the MARK-III convertor. Correction factors that take into account differences between the thermal and fast neutron spectrum as well as the spatial effects are described in the following section.

\section{RESUltS}

\section{A. U-235 Effective Mass}

The $\mathrm{M}_{\mathrm{eff}}{ }^{235}$ (impurities) of CFUL01 FCs, with U-238 as the main isotope in the deposit, were measured in the thermal flux of the BR1 empty cavity and were reported in [6]. In Table II the results of the $\mathrm{M}_{\mathrm{eff}}{ }^{235}$ that were reported in [6] are multiplied by $\mathrm{K}_{\mathrm{Meff}}{ }^{235}=1.090 \pm 0.033$ to fit to the MARK-III conditions 
because it was observed in earlier measurements with FCs of different designs that calibration in MARK-III leads to $9 \%$ bigger $\mathrm{M}_{\text {eff }}{ }^{235}$ than when calibrated in the empty cavity. This was confirmed in the current experiments (May 2021) with CFUL01 and CFUM21 FCs. The uncertainties of the results presented in Table II are propagated as discussed for this method in [7] and include the uncertainty on $\mathrm{K}_{\mathrm{Meff}}{ }^{235}$.

TABLE II

THE $\mathrm{M}_{\mathrm{EFF}}{ }^{235}$ IN CFUL01 FISSION CHAMBERS MEASURED IN BR1 EMPTY CAVITY AND MultiPLIED ON COEFFICIENT $\mathrm{K}_{\text {MeFF }}{ }^{235}=1.090$ TO FIT TO THE MARK-III CONDITIONS. UNCERTAINTIES ARE $3.6 \%$.

\begin{tabular}{|c|c|c|c|}
\hline \hline FC \# & $\mathbf{6 7 3}$ & $\mathbf{6 7 6}$ & $\mathbf{6 7 7}$ \\
\hline Dis. level & Meff $(\mu \mathrm{g})$ & Meff $(\mu \mathrm{g})$ & Meff $(\mu \mathrm{g})$ \\
$\mathbf{0 . 1 5 R}$ & 272 & 61 & 2020 \\
$\mathbf{0 . 2 R}$ & 258 & 60 & 1938 \\
$\mathbf{0 . 2 5 R}$ & 251 & 58 & 1885 \\
$\mathbf{0 . 3 R}$ & 243 & 57 & 1829 \\
$\mathbf{0 . 3 5 R}$ & 234 & 56 & 1770 \\
$\mathbf{0 . 4 R}$ & 224 & 54 & 1704 \\
$\mathbf{0 . 4 5 R}$ & 215 & 53 & 1633 \\
$\mathbf{0 . 5 R}$ & 204 & 51 & 1558 \\
$\mathbf{0 . 5 5 R}$ & 192 & 49 & 1478 \\
$\mathbf{0 . 6 R}$ & 179 & 46 & 1393 \\
\hline \hline
\end{tabular}

The $\mathrm{M}_{\text {eff }}{ }^{235}$ presented in Table II depends on the discrimination level R, which also can be seen in Fig.1.

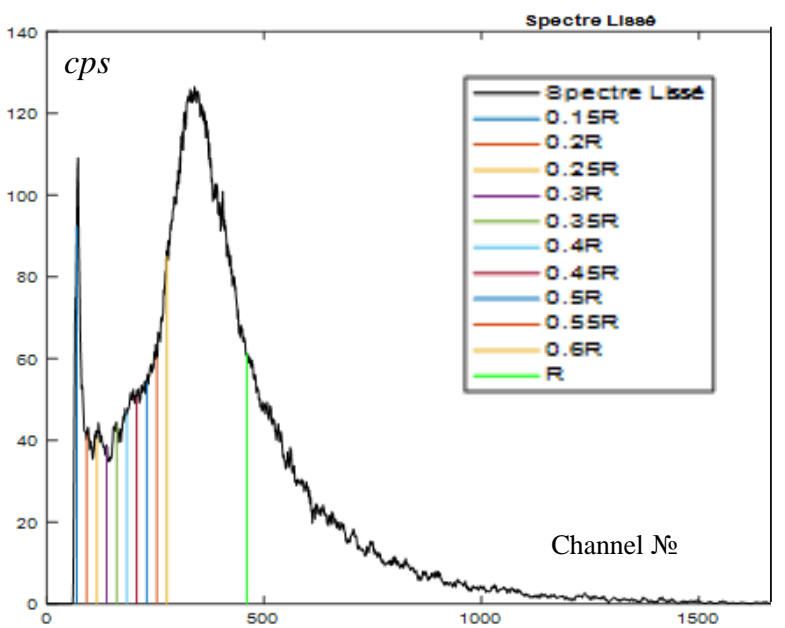

Fig. 1. Spectrum of U-235 fragments obtained with FC \#676 in the BR1 empty cavity.

\section{B. U-238 Effective Mass}

It is not straightforward to measure the $\mathrm{M}_{\text {eff }}{ }^{238}$ of CFUL01 in the fast flux of the MARK-III convertor because of its size. The length of the sensitive deposit $(211 \mathrm{~mm})$ is longer than the U235 convertor ( $80 \mathrm{~mm}$, see Fig. 2 ). Moreover, there is a gradient of the fission rates in the radial direction. [3]. Therefore the $\mathrm{M}_{\mathrm{eff}}{ }^{238}$ of CFUL01 measured in MARK-III should be corrected for these spatial effects. The coefficient $\mathrm{K}_{\text {Meff-238 }}{ }^{\mathrm{H}, \mathrm{R}}=1.787 \pm$ 0.036 (the uncertainty include statistics and the systematic contributions), which is the ratio of the $\mathrm{U}-238$ fission rate $(\mathrm{F} 28)$ in the centre of the MARK-III convertor without presence of the fission chamber to F28 in the real fission chamber deposits, was obtained with MCNP6 [8].

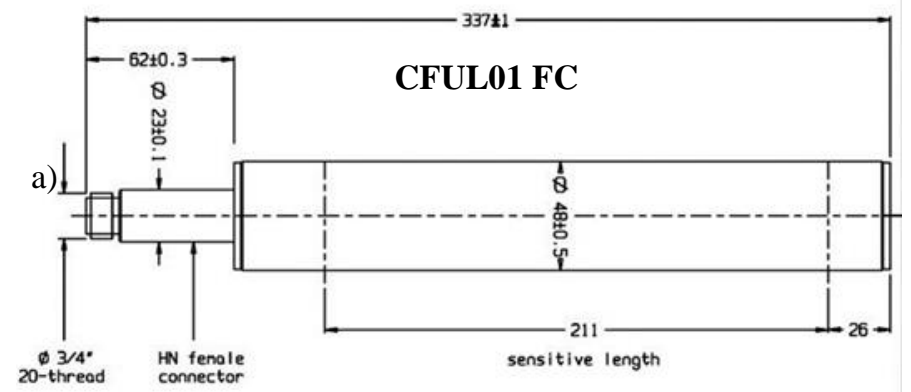

b)

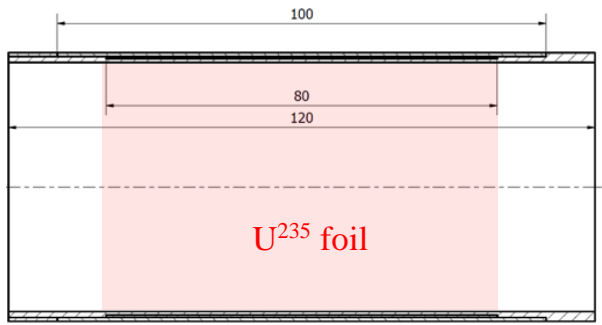

c)

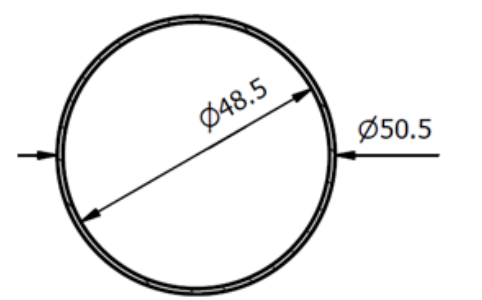

Fig. 2. The dimensions of the CFUL01 PHOTONIS FC (a) and the MARK-III U-235 90\% convertor in vertical (b) and horizontal (c) view.

The calculated coefficient $\mathrm{K}_{\text {Meff-238 }}{ }^{\mathrm{H}, \mathrm{R}}$-was verified with additional measurements and calculations of fission rates in axial F28(H) and radial F28(R) directions of the MARK-III convertor. The results are in a good agreement (see Fig. 3 and Fig.4). These results confirm the calculated coefficient $\mathrm{K}_{\text {Meff- }}$ ${ }_{238}^{\mathrm{H}, \mathrm{R}}$.

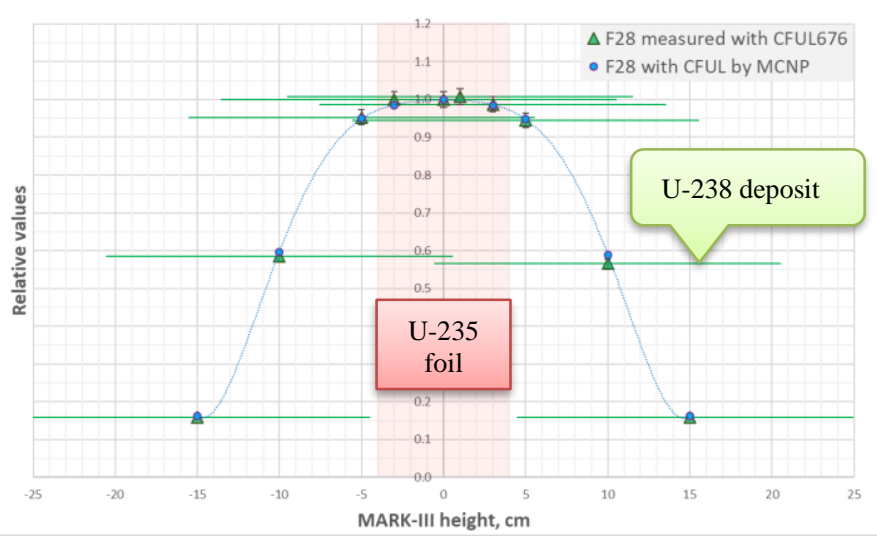

Fig. 3. Comparison of F28(H) measured with CFUL676 with F28(H) in CFUL01 calculated with MCNP in MARK-III. 


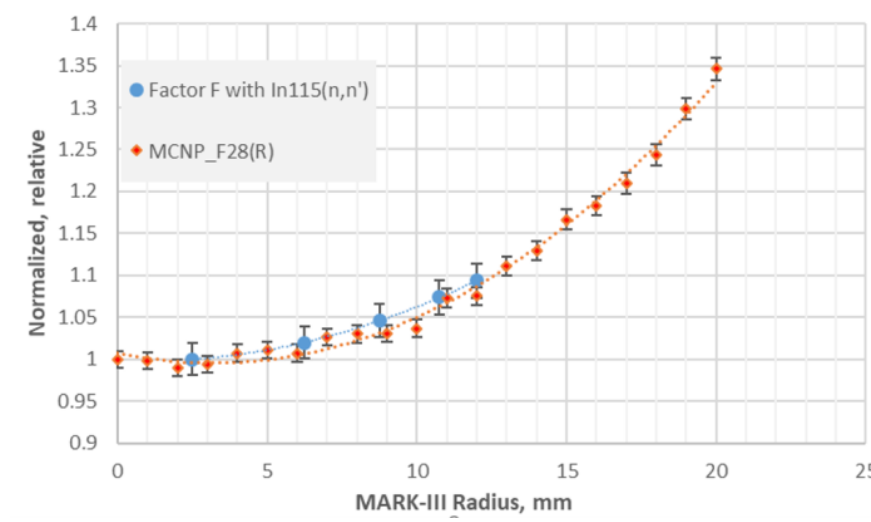

Fig. 4. Radial gradient in the MARK-III convertor (at axial position $\mathrm{H}=0 \mathrm{~cm}$ ) obtained with irradiation of $\operatorname{In}^{115}[3]$ and F28(R) calculated with MCNP.

The results of Meff ${ }^{238}$ of CFUL01 measured in the MARKIII fast neutron flux using (1) and multiplied by $\mathrm{K}_{\text {Meff- } 238}{ }^{\mathrm{H}, \mathrm{R}}$ are shown in Table III. The uncertainties of the results are $\pm 2.9 \%$ and they are propagated as discussed for this method in [6] and include the uncertainty on $\mathrm{K}_{\text {Meff-238 }}{ }^{\mathrm{H}, \mathrm{R}}$.

TABLE III

THE $\mathrm{M}_{\mathrm{EF}}^{238}$ IN CFUL01 FISSION CHAMBERS MEASURED IN MARK-III CONVERTOR AND MULTIPLIED ON COEFFICIENT $\mathrm{K}_{\text {MEFF-238 }}^{\mathrm{H}, \mathrm{R}}=1.787$.

\begin{tabular}{|c|c|c|c|}
\multicolumn{4}{c|}{ UNCERTAINTIES ARE 2.9\% } \\
\hline \hline FC \# & $\mathbf{6 7 3}$ & $\mathbf{6 7 6}$ & $\mathbf{6 7 7}$ \\
\hline Dis.level & Meff $(\mathrm{mg})$ & Meff $(\mathrm{mg})$ & Meff $(\mathrm{mg})$ \\
$\mathbf{0 . 1 5 R}$ & 712 & 854 & 912 \\
$\mathbf{0 . 2 R}$ & 706 & 814 & 893 \\
$\mathbf{0 . 2 5 R}$ & 685 & 791 & 864 \\
$\mathbf{0 . 3 R}$ & 662 & 766 & 838 \\
$\mathbf{0 . 3 5 R}$ & 638 & 740 & 809 \\
$\mathbf{0 . 4 R}$ & 612 & 712 & 779 \\
$\mathbf{0 . 4 5 R}$ & 584 & 681 & 745 \\
$\mathbf{0 . 5 R}$ & 552 & 648 & 710 \\
$\mathbf{0 . 5 5 R}$ & 518 & 613 & 672 \\
$\mathbf{0 . 6 R}$ & 479 & 574 & 631 \\
\hline \hline
\end{tabular}

\section{Isotopic Composition}

To obtain the enrichments or the impurities in the deposit of the fission chambers, the $\mathrm{M}_{\mathrm{eff}}{ }^{238}$ shown in Table III or the $\mathrm{M}_{\text {eff }}{ }^{235}$ presented in Table II were divided by the sum $\mathrm{M}_{\mathrm{eff}}{ }^{238}+$ $\mathrm{M}_{\text {eff }}{ }^{235}$. Since the $\mathrm{M}_{\text {eff }}$ depends on the discrimination level $\mathrm{R}$, for each ratio (to obtain the enrichment or impurity) the $\mathrm{M}_{\mathrm{eff}}{ }^{238}$ and $\mathrm{M}_{\text {eff }}{ }^{235}$ of the same $\mathrm{R}$ were taken. The results of the enrichments and the impurities obtained with the different discrimination levels are very close to each other (standard deviation when averaging $<0.1 \%$ ). The mean values are presented in Table IV and Table $\mathrm{V}$. The uncertainties in these tables are experimental ones (including of the uncertainty on the corrections obtained with MCNP) without the uncertainty on the certificate values because these are not available.

TABLE IV

MEASURED AND CERTIFICATE U-238 ENRICHMENT (IN \%).

\begin{tabular}{|l|c|c|c|c|}
\hline \hline FC\# & Certificate $(\mathrm{C}), \%$ & Measured $(\mathrm{E}), \%$ & $\pm \Delta$, abs. & C-E, \% \\
\hline $\mathbf{6 7 3}$ & 99.9650 & 99.9633 & 0.0028 & 0.0017 \\
$\mathbf{6 7 6}$ & 99.9988 & 99.9924 & 0.0006 & 0.0064 \\
$\mathbf{6 7 7}$ & 99.7984 & 99.7818 & 0.0168 & 0.0166 \\
\hline \hline
\end{tabular}

TABLE V

MEASURED AND CERTIFICATE U-235 IMPURITIES (IN \%)

\begin{tabular}{|l|c|c|c|c|}
\hline \hline FC\# & Certificate (C), \% & Measured $(\mathrm{E}), \%$ & $\pm \Delta, \mathrm{rel} \%$ & $\mathrm{C} / \mathrm{E}$ \\
\hline $\mathbf{6 7 3}$ & 0.0350 & 0.0367 & 3.8 & 0.95 \\
$\mathbf{6 7 6}$ & 0.0012 & 0.0076 & 3.8 & 0.16 \\
$\mathbf{6 7 7}$ & 0.2016 & 0.2182 & 3.8 & 0.92 \\
\hline \hline
\end{tabular}

\section{CONCLUSIONS}

Three PHOTONIS FCs of CFUL01 type with U-238 deposit, which were used in VENUS-F sub-critical experiments for beam interruption method validation, were calibrated in the BR1 reactor because the method was found to be sensitive to impurities in the deposits.

The enrichments and impurities of the deposits were obtained by measuring the effective masses of U-235 and U-238 in the accurately characterized thermal and fast neutron fields of the $\mathrm{BR} 1$ reactor.

In particular it was found that:

- $\quad$ All the measured results of U-238 enrichment in FCs are smaller than declared in their certificates.

- The declared U-235 impurities of two FCs are in agreement with the measurement results within $2 \sigma$. The $\mathrm{U}-235$ content in the third FC is 6 times bigger, which indicates a possible contamination of the base material in the deposit.

It is possible to measure Meff, impurities and enrichments of big PHOTONIS detectors like CFUL01 in the BR1 reactor.

\section{ACKNOWLEDGMENT}

This work was initiated as a prolongation of the verification of the sub-criticality monitoring for ADS MYRRHA which was carried out in European Projects FREYA FP7 (2011-2016), MYRTE H2020 (2016-2017) and CNRS $\backslash S C K \cdot C E N$ Project MYRACL (2017-2019).

\section{REFERENCES}

[1] J.-L. Lecouey et al., "Reactivity measurements with threshold fission chambers in the MYRRHA mock-up fast reactor VENUS-F", in Proc. PHYSOR 2018, Cancun, Mexico, April 22-26, 2018.

[2] J. Wagemans, E. Malambu, and L. Borms, "The Neutron Standard Fields at the BR1 Reactor at SCK•CEN," Journal of ASTM International 9, no. 3 (2012): 1-7

[3] L. Borms, "Characterisation of the fast neutron field in the BR1 MARK3 converter", Mol, Belgium, Internal report SCK•CEN, I-287, May 2015

[4] J. P. Hudelot et al., "Calibration des chambres à fissions EOLE/MINERVE et MASURCA dans les spectres de reference du reacteur BR1 du CEN/MOL", Cadarache, France, NT/DER/SPEx/LPE/02-037, 02.12.2002

[5] OECD/NEA Data Bank, "The JEFF-3.1.1 Nuclear Data Library", JEFF Report 22, Paris, 2009.

[6] A. Kochetkov et al., "Result of the tests of the new PHOTONIS CFUL01 fission chambers with high-purity U-238 deposit and $\mathrm{Al}$ containers with boron nitride cover", Mol, Belgium, SCK•CEN Report, R-6459, September 2017

[7] Adimir Dos Santos, "Guide to the expression of uncertainty", International Reactor Physics Experiments Evaluation Project (IRPhEP), Paris, OECD Report, NEA/NSC/DOC(2017), 2017

[8] C. J. Werner, MCNP User's manual Code version 6.2, LA-UR-1729981, 2017. 